\title{
THE CONSERVATION EQUATIONS FOR A MAGNETICALLY CONFINED GAS CORE NUCLEAR ROCKET
}

\author{
Terry Kammash and David L. Galbraith \\ Department of Nuclear Engineering \\ The University of Michigan \\ Ann Arbor, MI 48109
}

(313) 764-0205

\begin{abstract}
A very promising propulsion scheme that could meet the objectives of the Space Exploration Initiative (SEI) of sending manned missions to Mars in the early part of the next century is the open-cycle Gas Core (GCR) Nuclear Rocket. Preliminary assessments of the performance of such advice indicate that specific impulses of several thousand seconds, and thrusts of hundreds of kilonewtons are possible. These attractive propulsion parameters are obtained because the hydrogen propellant gets heated to very high temperatures by the energy radiated from a critical uranium core which is in the form of a plasma generated under very high pressure. Because of the relative motion between the propellant and the core, certain types of hydrodynamic instabilities can occur, and result in rapid escape of the fuel through the nozzle. One effective way of dealing with this instability is to place the system in an externally applied magnetic field. In this paper we formulate the appropriate conservation equations that describe the dynamics of GCR in the presence of magnetic fields, and indicate the role such fields play in the performance of the system.
\end{abstract}

\section{INTRODUCTION}

One of the most promising approaches to advanced space propulsion that could meet the objectives of the Space Exploration Initiative (SEI) is the open-cycle Gas Core Nuclear Rocket (GCR) (Ragsdale 1990) shown in Figure 1. The principle of operation in this system involves a critical uranium core in the form of a gaseous plasma that heats, through radiation, a seeded hydrogen propellant which exits through a nozzle, thereby converting thermal energy into thrust as illustrated in Figure 1. The hydrogen propellant is injected through a porous wall with a flow distribution that creates a relatively stagnant, non-recirculating central fuel region in the cavity. It has been found, on the basis of a heat transfer model (Poston and Kammash 1992) that takes into account the wall material temperature and heat

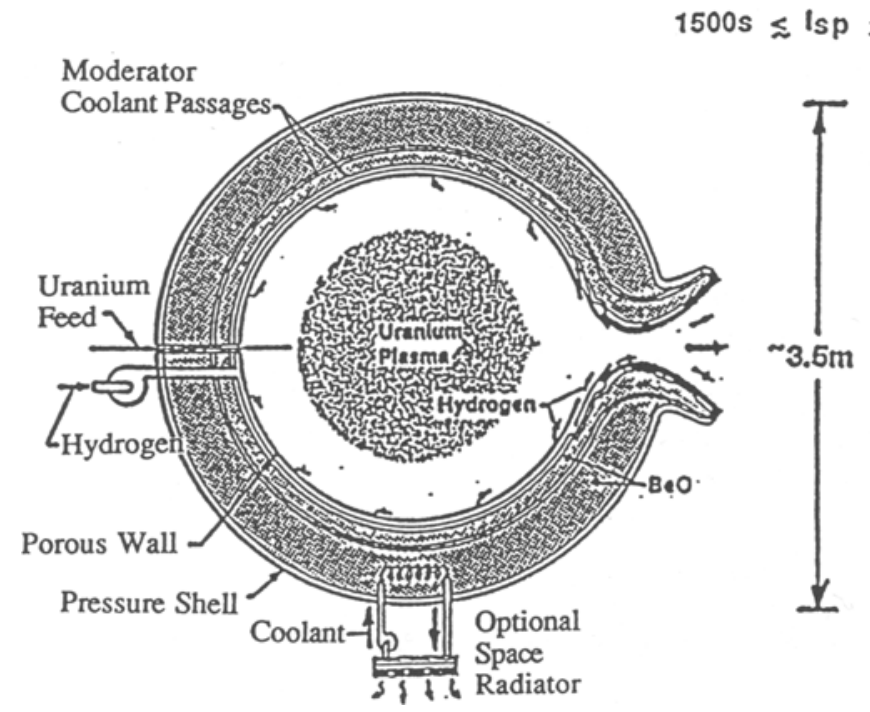

FIGURE 1. High Specific Impulse, Porous Wall Gas Core Engine (Courtesy of NASA, Lewis Research Center). 
flux, that specific impulses substantially less than the $5000 \mathrm{~S}$, often mentioned, are obtained when a maximum heat flux of $100 \mathrm{MW} / \mathrm{m}^{2}$ is imposed. Moreover, the relative motion between the hydrogen and the nearly stationary uranium core gives rise to the well known Kelvin-Helmholtz instability (Chandrasekhar 1961) which, if allowed to arise, could result in the loss of approximately $3 \%$ of the fuel per second (Kammash and Galbraith 1992). It has been found (Northrop 1956), however, that such an instability can be suppressed by placing the system in an externally-applied magnetic field in the direction of the propellant flow which acts as a "surface tension" type of force that provides the desired stability. Clearly, the configuration of such a field must be such that it confines the uranium core and yet allow the propellant to exhaust through the nozzle. The most logical field topology is that of a "mirror geometry" (Kammash 1975) in which the field is stronger at the ends than it is at the center where the uranium core is to be situated. The ratio of the field strength at the "mirrors," where the plasma particles are reflected, to that at the center is referred to as the mirror ratio, $R_{m}$. The confinement of charged particles in such a magnetic configuration is often expressed in terms of the confinement parameter $n \tau$ given by (Post 1987)

$$
n \tau \cong 2.5 \times 10^{10} E^{3 / 2} \log R_{m}
$$

where $\mathrm{n}$ is the density in $\mathrm{cm}^{-3}, \tau$ is the confinement time in seconds, and $\mathrm{E}$ is the mean energy of the particles in $\mathrm{keV}$. It is clear that perfect confinement requires an infinite mirror ratio (which is impossible), but significant reduction in the losses can be affected by moderate and technologically feasible values of $R_{m}$. The mirror ratio in effect defines a "loss cone" in velocity space which if, as a result of collisions with other charged particles, a particle falls in it, it will escape. It is clear, therefore, that geometric location of the particle in the system has no bearing on its confinement; rather it is the change in its velocity vector resulting from a collision with another particle that could place it in the loss cone and allow it to escape. For that reason it is reasonable to describe the system with a set of equations that ignore spatial variations. Such a set of conservation equations is often referred to as the pointreactor model which we will utilize to study the magnetically-confined gas core nuclear rocket.

\section{The Point-Reactor Model of a Magnetized GCR}

The containment of the uranium plasma in GCR by a mirror-type magnetic field, and assessment of its dynamic performance can be evaluated by writing an appropriate set of particle and energy conservation equations for the constituents of the core. The species under consideration are the uranium and fission fragment ions, and the electrons. The equations of interest are the particle equation for the fuel ions

$$
\frac{d N_{u}}{d \tau}=S_{u}-\frac{N_{u}}{\tau_{u}}-S_{F F}
$$

the energy balance equation for the fuel ions

$$
\frac{d\left(N_{u} E_{u}\right)}{d t}=S_{u} E_{i n}-\frac{N_{u}}{\tau_{u}} E_{L u}-S_{F F} E_{u}+N_{u}\left\{N_{e} W_{e u}+N_{F} W_{F u}+W_{F F u}\right\},
$$

the particle balance equation for thermalized fission fragments

$$
\frac{d N_{F}}{d t}=\frac{N_{F F}}{\tau_{F F}}-\frac{N_{F}}{\tau_{F}}
$$

the energy balance equation for thermalized fission fragments

$$
\frac{d\left(N_{F} E_{F}\right)}{d t}=\frac{N_{F F} E_{F}}{\tau_{F F}}-\frac{N_{F}}{\tau_{F}} E_{L F}-N_{F}\left\{N_{u} W_{F u}+N_{e} W_{F e}-W_{F F F}\right\},
$$


and the energy balance equation for the electrons

$$
\frac{d\left(N_{e} E_{e}\right)}{d t}=N_{e}\left\{N_{F} W_{F e}-N_{u} W_{e u}+W_{F F e}\right\}-\frac{N_{e}}{\tau_{e}} E_{L e}-P_{B},
$$

where $N_{u}$ is the number density of the uranium ions, $E_{u}$ is the energy of these ions, and $S_{u}$ is the source term for these particles. In a similar manner we define $N_{F}$ and $E_{F}$, and $N_{e}$ and $E_{e}$ for the fission fragments and the electrons respectively. The other terms are defined as follows: $\tau_{\mathrm{u}}$ is the confinement time for the uranium ions, and $\tau_{\mathrm{F}}$ and $\tau_{\mathrm{e}}$ are those for the fission fragments and electrons respectively. We distinguish in this analysis between the "fast" fission fragments whose density is designated by NFF and slowing down time by $\tau_{\mathrm{FF}}$, and the "thermalized" ones noted earlier. In the energy equations of the various species we note the terms designated by $\mathrm{W}$ with appropriate subscripts. These denote the rate of energy exchange between the species represented by the subscripts (Galbraith and Kammash 1978); for example, WFU represents the energy transfer rate from the thermal fission fragments to the uranium ions as a result of collisions, while $W_{\mathrm{FFU}}$ is the equivalent term for the exchange between the fast fission fragments and the uranium ions as these fragments slow down to reach thermalization. In Equation (2) the term $S_{\mathrm{FF}}$ represents the rate at which uranium ions are lost as a result of their undergoing fission reactions, and this term should serve as the source term for the fission fragments number density seen in Equation (4). The terms $\mathrm{E}_{\mathrm{Le}}, \mathrm{E}_{\mathrm{Lu}}$, and $E_{L F}$ represent the escape energy terms for the electrons, uranium ions, and fission fragment ions respectively, and are found from the mirror machine energy confinement equations (Galbraith and Kammash 1978). For the electrons, we assume that the time rate of change of their number density is much faster than that of the other species, that their particle balance equation may be replaced by a steady state equation of charge balance:

$$
\frac{N_{e}}{\tau_{e}}-\left(Z_{e f f}\right)_{u} \frac{N_{u}}{\tau_{u}}-\left(Z_{e f f}\right)_{F} \frac{N_{F}}{\tau_{F}}=0,
$$

where $\left(\mathrm{Z}_{\mathrm{eff}}\right)_{\mathrm{u}}$ denotes the effective charge on the uranium ions and a similar term for the fission fragments. It should be kept in mind that $S_{F F}=N_{u} \phi \sigma_{f}$, where $\phi$ is the neutron flux, and $\sigma_{\mathrm{f}}$ is the fission cross section for the uranium Moreover the quantity $\mathrm{N}_{\mathrm{e}}$ is found from the Saha equations which relate it to the densities and temperatures of the other species in the system (Kammash and Galbraith 1989). The electron confinement time $\tau_{e}$, and escape energy $E_{L e}$ are obtained using Pastukhov's equations (Galbraith and Kammash 1978), and these quantities along with $\tau_{\mathrm{u}}$ and $\mathrm{E}_{\mathrm{Lu}}$ and $\tau_{\mathrm{F}}$ and $\mathrm{E}_{\mathrm{LF}}$ require the ambipolar potential $\mathrm{e} \Phi$ which is obtained iteratively, at the same time as $\tau_{\mathrm{e}}$, by requiring that Equation (7) be satisfied. This electrostatic potential $\Phi$ is positive and is created by the deficiency in the negative charge in the system that arises from the rapid loss of the electrons through the mirrors. The term $P_{B}$ that appears in Equation (6) of the electron energy balance equation represents the radiative loss in the form of continuous $\mathrm{X}$-rays which we assume to escape completely from the core. Another radiative loss term, known as synchrotron radiation, is ignored in this analysis in spite of the presence of the magnetic field, since the plasma temperature for the case at hand is considered relatively low. The fission energy produced in the reactor appears in the fission fragments since the portion carried out by the neutrons and gamma rays is not accounted for by the equations presented here which deal exclusively with charged particles.

No attempt is made in this study to calculate particle and energy losses across the magnetic field so as to assess the extent of mixing of fuel particles with the propellant. Moreover no attempt will be made to present the solution of the governing equations formulated above at this time; rather some general comments will be put forward that will describe qualitatively the behavior of a critical uranium plasma situated in a mirror-type magnetic field. We note first that the energy produced by fission in the reactor, namely about $175 \mathrm{MeV}$ out of about $200 \mathrm{MeV}$, appears in the terms $W_{F F u}, W_{F F F}$, and $W_{F F e}$, the fast fission fragments which pass it on to the uranium ions, the thermal fission fragment ions, and the electrons as seen in Equations (3), (5), and (6). The energy of these particles increases as a result, and according to the confinement law, Equation (1), they will become better confined. Moreover, better confinement is obtained by increasing the mirror ratio, and if 54 Gauss is required at the center for stabilization, then about $5.5 \mathrm{KG}$ will be needed at the mirrors to generate a mirror ratio of 100 . For such a mirror ratio simple estimates indicate that over $90 \%$ of the particles remain confined. Since some losses are inevitable it is important that they occur through the mirror that coincides with the nozzle and not in the opposite direction. This can be accomplished by making the mirror machine slightly asymmetric by making the mirror ratio on the nozzle side slightly smaller than that at the opposite side. 


\section{BESULTS AND DISCUSSION}

Estimates of the uranium loss rate from this magnetic configuration can be obtained from a modified version of Equation (1) that takes into account the fact that the ions under consideration are uranium ions, and that the mirror becomes more lossy in the presence of an electrostatic potential $\Phi$ which is consistent with Equation (7). The resulting expression is

$$
\frac{N u}{\tau_{u}}=\left(3.844 \times 1-^{-12}\right) \frac{N_{u}^{2} Z_{e f f}^{2}}{E_{u}^{3 / 2} \log _{10}\left(\frac{R_{m}}{1+Z_{u} e \Phi / E_{u}}\right)}
$$

for a spherical GCR with $1 \mathrm{~m}$ radius and a fuel loading of $0.25 \mathrm{Kg}$ and a mirror ratio of $R_{\mathrm{m}}=100$. Table 1 shows the corresponding loss rates.

TABLE 1. Uranium loss rate from a magnetic mirror.

\begin{tabular}{cccc}
$\mathrm{E}_{\mathrm{u}}(\mathrm{keV})$ & $\mathrm{Z}_{\text {eff } \mathrm{u}}$ & $\mathrm{e} \Phi(\mathrm{keV})$ & $\mathrm{N}_{\mathrm{u}} / \tau_{\mathrm{u}}\left(\mathrm{cm}^{-3} \mathrm{sec}^{-1}\right)$ \\
\hline 0.005 & 3.831 & 0.0133 & $5.756 \times 10^{28}$ \\
0.050 & 18.001 & 0.0438 & $1.089 \times 10^{30}$ \\
1.000 & 77.817 & 0.1614 & $3.798 \times 10^{30}$ \\
10.000 & 91.106 & 1.0547 & $2.009 \times 10^{29}$
\end{tabular}

It is clear that these losses are prohibitively large, and that a magnetic mirror geometry as represented by Equation (8) may be inappropriate or not applicable. It is equally clear that the presence of a magnetic field should not worsen the confinement. The difficulty lies with Equation (8) since it assumes that a given particle makes, on the average, many transits of the core before suffering a collision. However, for the dense, highly charged uranium ions of GCR, the reverse is true. The transit time is $\tau_{t r}=r / c_{S}$ where $c_{S}$ is the thermal speed of the particle, and that should be compared with the $90^{\circ}$ collision time $\tau_{\theta}$ (Kammash 1975). The results are given in Table 2.

TABLE 2. Comparison of Collision and Transit Times.

\begin{tabular}{cccc}
$\mathrm{E}_{\mathbf{u}}(\mathrm{keV})$ & $\mathrm{Z}_{\text {eff } \mathbf{u}}$ & $\tau_{\mathrm{tr}}(\mathrm{sec})$ & $\tau_{\theta}(\mathrm{sec})$ \\
\hline 0.005 & 3.831 & $8.579 \times 10^{-4}$ & $9.491 \times 10^{-13}$ \\
0.050 & 18.001 & $2.731 \times 10^{-4}$ & $6.971 \times 10^{-14}$ \\
1.000 & 77.817 & $6.066 \times 10^{-5}$ & $1.325 \times 10^{-14}$ \\
10.000 & 91.106 & $1.918 \times 10^{-5}$ & $1.160 \times 10^{-13}$
\end{tabular}

These figures reveal, in a dramatic way, that a typical particle will undergo many scattering collisions before it transits out of the system. As a result, the uranium plasma in GCR does not behave like a nearly collisionless gas for which a confinement law such as Equation (8) is applicable; rather it behaves much like a dense fluid for which the collision mean free path is much smaller than any characteristic dimension of the system. Such a description entails the use of a different confinement law, for a magnetic mirror, to be used in the above-mentioned balance equations that will allow a more accurate description of the performance of the system.

\section{SUMMARY AND CONCLUSIONS}

The particle and energy balance equations presented in this paper to describe the dynamics of GCR uranium plasma situated in a mirror-type magnetic field are adequate provided they are used in conjunction with a confinement law that is appropriate for a high density, high charge plasma system. Such a law should show enhancement of confinement with mirror ratio and size but lack of enhancement with thermal velocity or temperature. 


\section{Acknowledgments}

This work was supported in part by the U. S. Department of Energy under Contract No. DE-FG02-93ER75869.

\section{References}

Chandrasekhar, S. (1961) Hydrodynamic and Hydromagnetic Stability, Dover Publications, New York.

Galbraith, D. L. and T. Kammash (1978) "Electron and Ion Escape over a Potential Barrier in a Mirror Field," Plasma Physics 20: 959.

Galbraith, D. L. and T. Kammash (1978) "The Dynamic Behavior of a Mirror Fusion Reactor," Electric Power Research Institute Report EPRI ER-521, March 1978.

Kammash, T. and D. L. Galbraith (1992) "Fuel Confinement and Stability in the Gas Core Nuclear Propulsion Concept," AJAA 92-3818, 28th Joint Propulsion Conference, Nashville, TN, 6-8 July 1992.

Kammash, T. and D. L. Galbraith (1989) "A High Gain Fusion Reactor Based on the Magnetically Insulated Inertial Confinement Fusion Concept," Nuclear Fusion 29: 1079.

Kammash, T. (1975) Fusion Reactor Physics, Principles and Technology, Ann Arbor Science Publishers, Ann Arbor, MI.

Northrop, T. G. (1956) "Helmholtz Instability of a Plasma," Physical Review 103: 1150.

Post, R. F. (1987) "The Magnetic Mirror Approach to Fusion," Nuclear Fusion 27: 1579.

Poston, D. I. and T. Kammash (1992) "Heat Transfer Model for an Open-Cycle Gas Core Nuclear Rocket," in Proc. Ninth Symposium on Space Nuclear Power Systems, M.S. El-Genk and M. D. Hoover, eds., American Institute of Physics, New York, AIP Conference Proceeding, No. 246: 1083-1088.

Ragsdale, R. G. (1990) Nuclear Thermal Propulsion, a Joint NASA/DOE/DOD Workshop, Cleveland, OH, 10-12 July 1990. 